\title{
DENSIDADE APARENTE MÉDIA DE RESÍDUOS SÓLIDOS COLETADOS EM UMA OBRA PORTUÁRIA
}

\author{
AVERAGE BULK DENSITY OF SOLID WASTE COLLECTED IN A PORT \\ CONSTRUCTION SITE
}

\section{DENSIDAD APARENTE PROMEDIO DE RESIDUOS SÓLIDOS RECOLECTADOS EN UNA OBRA PORTUARIA}

\author{
André Nagalli ${ }^{1}$ \\ Paulo Roberto Geraldo Filho ${ }^{2}$ \\ Natália Smaniotto Bach ${ }^{3}$
}

\section{Resumo}

As taxas de geração de resíduos sólidos na construção civil são variáveis e dependentes de diversos fatores. Obras de grande porte têm características distintas; desta forma, é importante dispor de indicadores de geração de resíduos para ações de planejamento e gestão. Neste trabalho, investigaram-se as densidades aparentes de alguns tipos de resíduos sólidos gerados em uma obra de construção portuária no município de Paranaguá, Paraná. Utilizou-se a pesagem das caçambas estacionárias como forma de aquisição de dados. Os resultados revelaram densidade aparente média variável por tipo de material: 0,605 t/m³ para resíduos classe A (Conama 307/02); 0,307 t/m³ para sucata de madeira; $0,696 \mathrm{t} / \mathrm{m}^{3}$ para sucata metálica; $0,132 \mathrm{t} / \mathrm{m}^{3}$ para rejeitos classe IIA; $0,083 \mathrm{t} / \mathrm{m}^{3}$ para sucata de papel e $0,087 \mathrm{t} / \mathrm{m}^{3}$ para sucata plástica. Observou-se que a escala de medição - caçamba estacionária versus bombona plástica - interfere diretamente nos resultados. Apesar das especificidades das grandes obras, concluise que os indicadores obtidos podem ser aplicados nesse tipo de empreendimento. Esses indicadores são, dessa forma, mais adequados do que os disponíveis atualmente na literatura.

Palavras-chave: Densidade aparente média. Resíduos sólidos. Construção civil.

\begin{abstract}
The rates of solid waste generation in civil construction are variable and dependent on several factors. Large works have distinct characteristics; thus, it is interesting to have indicators of waste generation for planning and management actions. In this work, we investigated the apparent densities of some types of solid waste generated in a port construction project in the municipality of Paranaguá, Paraná. The weighing of stationary buckets was used as a form of data acquisition. The results revealed average bulk density by material type: $0.605 \mathrm{t} / \mathrm{m}^{3}$ for class A residues (Conama 307/02); $0.307 \mathrm{t} / \mathrm{m}^{3}$ for scrap wood; $0.696 \mathrm{t} / \mathrm{m}^{3}$ for scrap metal; $0.132 \mathrm{t} / \mathrm{m}^{3}$ for class IIA tailings; $0.083 \mathrm{t} / \mathrm{m}^{3}$ for scrap paper and $0.087 \mathrm{t} / \mathrm{m}^{3}$ for plastic scrap. It was observed that the measurement scale stationary bucket versus plastic barrel - directly interferes with the results. It is concluded that, despite the particularities of the great works, the indicators obtained can be applied in large construction projects. These indicators are, therefore, more appropriate than those currently available in the literature.
\end{abstract}

Keywords: Average bulk density. Solid waste. Civil construction.

\section{Resumen}

Las tasas de generación de residuos sólidos en la construcción civil son variables y dependen de diversos factores. Las obras de grande porte tienen características distintas; por ello, es importante disponer de indicadores de producción de residuos para acciones de planificación y gestión. En este trabajo se estudiaron las densidades aparentes de algunos tipos de residuos sólidos producidos en una obra de construcción portuaria en el municipio

\footnotetext{
${ }^{1}$ Engenheiro Civil. Mestre em Engenharia de Recursos Hídricos e Ambiental. Doutor em Geologia. UTFPR - Universidade Tecnológica Federal do Paraná - UTFPR. E-mail: nagalli@utfpr.edu.br

${ }^{2}$ Especialização em Engenharia de Segurança do Trabalho. E-mail: engpauloroberto87@gmail.com.

${ }^{3}$ Engenheira Civil. UTFPR - Universidade Tecnológica Federal do Paraná - UTFPR. E-mail: natalia.smaniotto@hotmail.com.
} 
de Paranaguá, en el estado de Paraná. Se estudió el peso de los contenedores de escombros como forma de recolección de datos. Los resultados revelaron densidad aparente promedio variable, de acuerdo con el tipo de material: $0,605 \mathrm{t} / \mathrm{m}^{3}$ para residuos clase A (Conama 307/02); 0,307 t/m3 para residuos de madera; $0,696 \mathrm{t} / \mathrm{m}^{3}$ para residuos metálicos; $0,132 \mathrm{t} / \mathrm{m}^{3}$ para residuos clase IIA; $0,083 \mathrm{t} / \mathrm{m}^{3}$ para residuos de papel y $0,087 \mathrm{t} / \mathrm{m}^{3}$ para residuos plásticos. Se pudo observar que la escala de medición - contenedores de escombros versus cubos de basura plásticos - interfiere directamente en los resultados. A pesar de las especificidades de las grandes obras, se concluye que los indicadores obtenidos pueden ser aplicados en ese tipo de desarrollo. De esa manera, esos indicadores son más adecuados que los disponibles actualmente en la literatura.

Palabras-clave: Densidad aparente promedio. Residuos sólidos. Construcción civil.

\section{Introdução}

As palavras excesso de consumo, entulho e uso imprudente de materiais são associadas, regularmente, à construção civil. Entretanto, nas últimas décadas, esse setor tem se modernizado e buscado superar hábitos e modos de trabalho antigos.

A indústria da construção civil, grande geradora de empregos diretos e indiretos, possui significativa participação no desenvolvimento econômico mundial. Esse setor contribui de modo direto e significativo para o aumento do produto interno bruto, possuindo potencial para ser uma das esferas industriais mais dinâmicas no centro do crescimento econômico global (ONAT; KUCUKVAR, 2020). A indústria da construção global tem rápido crescimento decorrente do aumento dos investimentos nos setores de infraestrutura, construção, energia e transporte (ONAT; KUCUKVAR, 2020). Espera-se que o crescimento médio da construção seja de $67 \%$ em todo o mundo até 2020, o que representa um avanço de 5,2\% ao ano (BETTS et al., 2009).

Com base na previsão global de construção para 2020, China, Canadá, Índia, Japão e EUA devem ser os principais contribuintes para a indústria da construção global em todo o mundo (BETTS et al., 2009).

Em países desenvolvidos, a indústria da construção é responsável por 25 a $30 \%$ da quantidade total de resíduos descartados (LU et al., 2017). Estima-se que o volume de resíduos de construção e demolição (RCC) no Brasil represente, aproximadamente, 67\% do total de resíduos sólidos urbanos (RSU) gerados no país (SCHAMNE; NAGALLI, 2018). Esta situação decorre da baixa eficiência do processo de gerenciamento dos RCC, o que causa o tratamento e disposição inadequados dos resíduos (SCHAMNE; NAGALLI, 2018); nesse sentido, pesquisas sobre resíduos de construção e demolição vêm aumentando significativamente (YUAN, 2013).

Em ambientes urbanos, a geração de resíduos de construção e demolição é inevitável; logo, durante a consolidação das cidades e nos períodos de expansão da população, os esforços para realizar uma gestão mais criteriosa do setor da construção e de demolição devem ser 
permanentes (DE MELO et al., 2011).

Alguns fatores que contribuem com a geração de entulho são: projetos com falta de detalhamentos e especificações, materiais com pouca qualidade que são adotados pelo baixo custo, falta de cuidado com armazenamento e transporte de materiais dentro e fora da obra, falta de mão-de-obra qualificada, pouco controle e fiscalização dos processos executivos, entre outros.

O conhecimento do índice de resíduos gerados é importante para definir uma estratégia de gestão de resíduos; este permite, por exemplo, definir o tamanho dos recipientes de coleta, a melhor forma de transporte interno e externo, os potenciais para reaproveitamento e pare reciclagem de resíduos, ou seja, a logística de manejo dos resíduos (NAGALLI, 2014).

Neste contexto, ressalta-se a necessidade de estudos e ferramentas que auxiliem na quantificação de resíduos sólidos a serem gerados em todos os tipos de obras, considerando-se as diversas variáveis que influenciam na geração de resíduos sólidos e, consequentemente, nas suas densidades (PAZ, 2019).

A logística de coleta de detritos pressupõe compreender a quantidade de resíduos que será gerada durante a obra e, consequentemente, qual estrutura necessária para o acondicionamento e armazenagem destes. Dentre os aspectos que afetam o dimensionamento desta estrutura, está a compreensão da densidade aparente dos resíduos gerados. No setor portuário, a gestão de resíduos de construção ainda é pouco estudada, não tendo sido evidenciados estudos similares na literatura.

O presente trabalho quantificou a densidade aparente média de seis tipos de resíduos sólidos: resíduos da construção civil (RCC), madeira, metal, resíduo orgânico, papel e plástico. A análise foi realizada em uma obra de engenharia portuária situada no município de Paranaguá, Estado do Paraná.

\section{Metodologia}

O método de pesquisa incluiu o levantamento de dados de pesagem de resíduos a partir de Manifestos de Transporte de Resíduos (MTR) disponibilizados pela empresa construtora da obra portuária. Este é um documento que se presta a controlar a expedição, o transporte e o recebimento dos resíduos ao longo de seu ciclo, sendo caracteristicamente emitido pelos transportadores. Foram analisados dados de um período de 10 meses de trabalho.

A obra de infraestrutura portuária cujos resíduos foram estudados é a de construção do berço 218, dolphins e retroárea do berço 217, no extremo Leste do Terminal de Contêineres de 
Paranaguá (TCP), no município de Paranaguá, Paraná. Trata-se uma obra portuária completa, que abrangeu serviços habituais de canteiros de serviços (carpintaria, central de armação, usinagem de concreto, refeitório, ambulatório, escritórios, etc.), produção de peças prémoldadas em concreto para utilização na obra, cravação de estacas/camisas metálicas, de estacas em concreto, com balsas, com guindastes por terra, etc., além de serviços correlatos.

Os valores de densidade aparente média dos resíduos sólidos foram calculados a partir das massas de resíduos obtidas por pesagem em balanças tipo rodoviária. Analisaram-se 10 MTR's (registros) para cada um dos seis tipos de resíduos estudados: resíduos de construção civil classe A (Resolução Conama 307/02), sucata de madeira, sucata metálica, rejeitos classe IIA, sucata de papel e sucata plástica (ASSOCIAÇÃO BRASILEIRA DE NORMAS TÉCNICAS, 2004).

Os resíduos foram acondicionados em caçambas estacionárias pela equipe responsável pela limpeza e conservação do canteiro de obras, padronizadas com volumes de $5 \mathrm{~m}^{3}$ para sucata de madeira, sucata metálica, rejeitos classe IIA, sucata de papel e sucata plástica. Para o RCC classe A foram utilizadas caçambas roll on - roll off de $25 \mathrm{~m}^{3}$.

\section{Resultados}

As massas dos resíduos identificadas estão apresentadas na Tabela 1.

Tabela 1: Massa dos resíduos gerados (Pesagem registrada nos MTRs)

\begin{tabular}{lcccccc}
\hline & \multicolumn{5}{c}{ Tipo de Resíduo } \\
\cline { 2 - 7 } & RCC & Sucata de & Sucata & Rejeito & Sucata de & Sucata \\
& Classe A & Madeira & Metálica & Classe IIA & Papel & Plástica \\
\hline & 13,38 & 1,35 & 2,73 & 0,67 & 0,50 & 0,40 \\
& 14,90 & 1,50 & 3,12 & 0,65 & 0,20 & 0,50 \\
& 18,21 & 1,45 & 2,75 & 0,49 & 0,30 & 0,45 \\
Massa de & 12,79 & 1,70 & 3,24 & 0,54 & 0,35 & 0,35 \\
resíduos em & 20,21 & 1,80 & 3,62 & 0,50 & 0,35 & 0,50 \\
cada caçamba & 13,46 & 1,80 & 3,93 & 0,65 & 0,85 & 0,40 \\
(t) & 16,25 & 1,60 & 6,88 & 0,54 & 0,32 & 0,50 \\
& 13,86 & 1,50 & 6,47 & 0,90 & 0,30 & 0,45 \\
& 14,45 & 1,30 & 1,31 & 0,68 & 0,50 & 0,40 \\
& 13,75 & 1,35 & 0,76 & 1,00 & 0,51 & 0,40 \\
\cline { 2 - 7 } Média (t) & $\mathbf{1 5 , 1 3}$ & $\mathbf{1 , 5 4}$ & $\mathbf{3 , 4 8}$ & $\mathbf{0 , 6 6}$ & $\mathbf{0 , 4 2}$ & $\mathbf{0 , 4 4}$ \\
Desvio Padrão & $\mathbf{2 , 4 0}$ & $\mathbf{0 , 1 8}$ & $\mathbf{1 , 9 5}$ & $\mathbf{0 , 1 7}$ & $\mathbf{0 , 1 8}$ & $\mathbf{0 , 0 5}$ \\
\hline
\end{tabular}

Fonte: Autor 
Da análise da Tabela 1, depreende-se que não há constância nos valores de pesagem, o que sugere que cada carga de resíduos possui características próprias e não homogeneidade. Nota-se que a maior variação (56\%) é observada no resíduo sucata metálica, enquanto as menores variações (12\%) são observadas nas categorias sucata de madeira e sucata plástica. Todavia, considerando-se os desvios padrão calculados, pode-se afirmar que, em geral, os valores médios obtidos são válidos para fins de gestão de obras. Faz-se uma ressalva ao indicador obtido para a sucata metálica, cujo desvio padrão é considerado alto (1,95 comparado à média de 3,48). Atribui-se a variação dos resultados das pesagens ao tipo e forma de resíduo/material acondicionado, geometria dos resíduos, grau de compactação, massa específica, umidade e aspectos correlatos.

Para determinar se a densidade aparente média $\left(\mathrm{t} / \mathrm{m}^{3}\right)$ dos diversos tipos de resíduos sólidos, fez-se a relação entre a massa da amostra e o volume da caçamba estacionária que acondicionou os detritos. A Figura 1 apresenta os resultados calculados para as densidades aparentes de cada resíduo.

Figura 1: Médias das densidades aparentes médias dos resíduos estudados

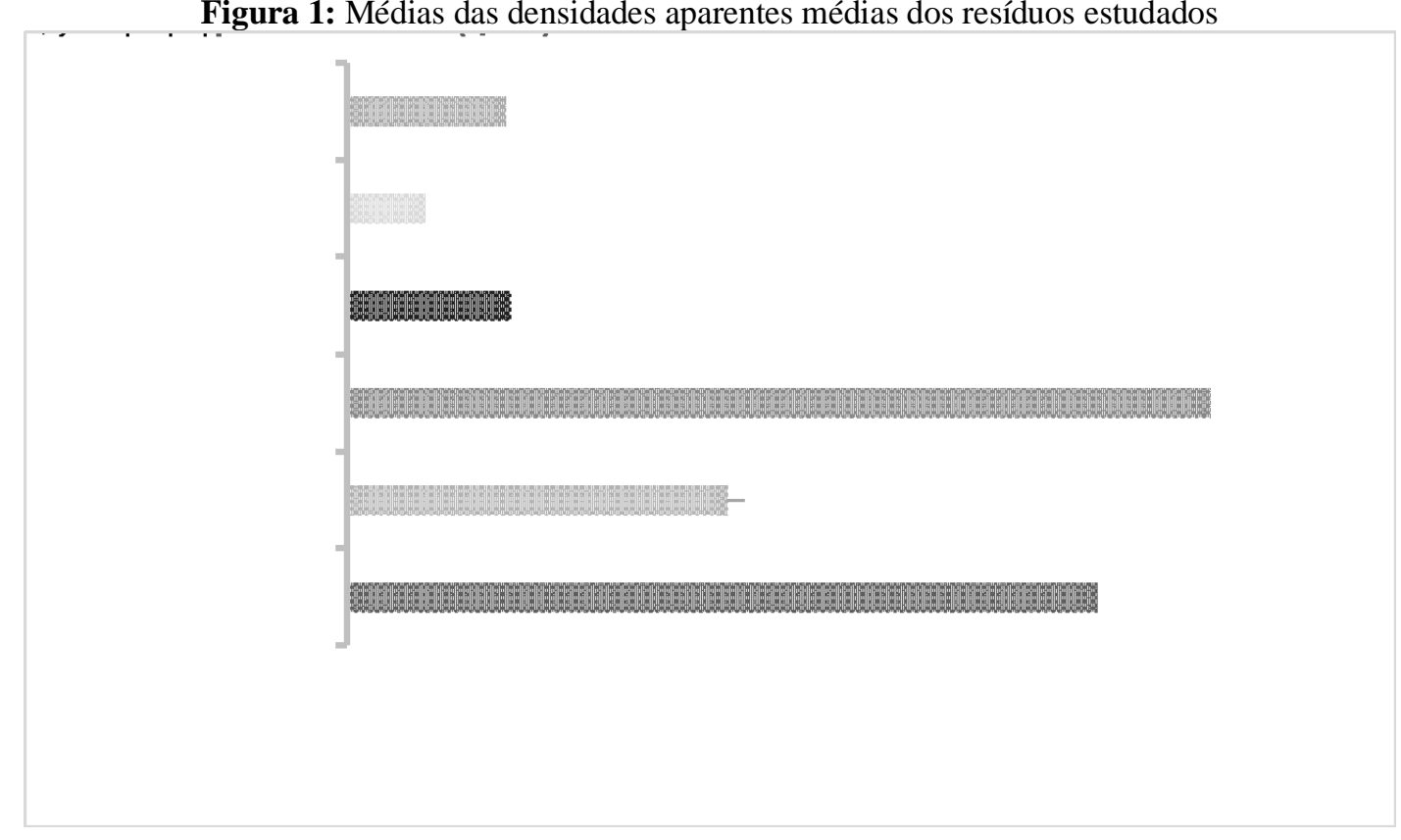

Da análise da Figura 1, observa-se que os resíduos com maior densidade aparente são a sucata metálica e o resíduo de construção civil classe A, o que aponta um resultado esperado; já os resíduos de menor densidade aparente são a sucata de papel e a sucata de plástico. Os resultados foram comparados com a literatura: Vasconcelos e Lemos (2015) e Silva e Santos (2011) realizaram estudos com o intuito de estimar a densidade aparente média de alguns materiais coletados na região de Belo Horizonte e de Fortaleza, respectivamente, e obtiveram 
os valores apresentados na Tabela 2.

Tabela 2: comparativo dos resultados obtidos com a literatura

Densidade aparente $\left(t / \mathbf{m}^{3}\right)$

\begin{tabular}{lccc}
\hline Tipo de Resíduo & Resultados & $\begin{array}{c}\text { Vasconcelos e Lemos } \\
(\mathbf{2 0 1 5})\end{array}$ & Silva e Santos (2011) \\
\hline RCC classe A & $\mathbf{0 , 6 0 5}$ & - & 0,240 \\
Sucata de Madeira & $\mathbf{0 , 3 0 7}$ & 0,140 & 0,041 \\
Sucata Metálica & $\mathbf{0 , 6 9 6}$ & 0,253 & 0,053 \\
Rejeitos Classe IIA & $\mathbf{0 , 1 3 2}$ & - & - \\
Sucata de Papel & $\mathbf{0 , 0 8 3}$ & 0,058 & 0,338 \\
Sucata Plástica & $\mathbf{0 , 0 8 7}$ & 0,043 & 0,135 \\
\hline
\end{tabular}

Da análise da Tabela 2, depreende-se que os resultados obtidos por esse estudo através de aferições com caçambas estacionárias de grande capacidade $\left(5 \mathrm{~m}^{3}\right)$ - tendem a apresentar valores distintos dos observados por pesquisas anteriores. Além das características dos resíduos, a medição também pode ser influenciada pela capacidade do recipiente de medição, pois estudos anteriores utilizaram receptáculos de escala inferior 150L em Vasconcelos e Lemos (2015) e 250kg em Silva e Santos (2011).

Pela observação da Tabela 2, constata-se, ainda, que não há um padrão de valores, pois a densidade aparente variável é dependente de diversos fatores. A grande variabilidade é atribuída não somente às características distintas dos materiais e às formas de acondicionamento, mas também à escala de medição — conforme argumento anterior.

Neste contexto, confirma-se a necessidade de investir em novas pesquisas e coletas de informações que possibilitem aos projetistas antever as estruturas necessárias para o correto gerenciamento dos resíduos na construção civil. Constatou-se que na obra portuária analisada os resíduos de construção apresentam densidade aparente bastante diversa entre si; esse contexto demanda uma logística de coleta dinâmica e estrutura de coleta adaptável, já que a velocidade de "enchimento" dos contentores de resíduo tende a ser variável, postas as taxas de geração e densidades aparentes diversificadas.

Por outro lado, obras de grande porte costumam ter similaridades quanto à geração de resíduos, uma vez que dispõem de estruturas complexas (refeitórios, ambulatórios, grande quantidade de sanitários e escritórios, carpintaria, central de armação, etc.). Considerando as altas taxas de geração, é usual que tais obras disponham de estruturas com caçambas 
estacionárias de grande capacidade para coleta de resíduos, o que monta às densidades aparentes medidas, invariáveis ao tipo de obra, em extrapolação hipotética. Desta forma, embora os indicadores obtidos possam se modificar, tais variáveis constituem-se em importante procedimento preliminar de planejamento e de gestão de resíduos; logo, durante seu funcionamento, esse sistema de gestão deve ser constantemente monitorado e adaptado às circunstâncias.

\section{Conclusão}

Os resultados permitiram concluir que a densidade aparente média dos resíduos sólidos é variável e está diretamente ligada às características físicas de cada material. Os valores de densidade média aparente encontrados na obra portuária analisada foram os seguintes: 0,605 t/m³, para resíduos de construção civil classe A (Conama 307/02); 0,307 t/m³, para sucata de madeira; $0,696 \mathrm{t} / \mathrm{m}^{3}$, para sucata metálica; $0,132 \mathrm{t} / \mathrm{m}^{3}$, para rejeitos classe IIA (ASSOCIAÇÃO BRASILEIRA DE NORMAS TÉCNICAS, 2004); 0,083 t/m³ , para sucata de papel; e 0,087 $\mathrm{t} / \mathrm{m}^{3}$, para sucata plástica.

A comparação desses resultados com os dados da literatura demonstrou que há grande variação na densidade aparente dos resíduos sólidos, o que pode ser atribuído às formas de acondicionamento, grau de compactação, características físicas dos resíduos e tipos de obras. Verificou-se, além disso, que os resultados de densidade aparente são afetados pelo tamanho do recipiente de coleta/medição. Desta forma, é essencial a continuidade de pesquisas na área para a produção de novos indicadores de geração que sejam adaptáveis à realidade de cada obra.

Por outro lado, é possível aplicar os indicadores obtidos nesta pesquisa no planejamento da estrutura de gestão de resíduos sólidos em futuras obras de infraestrutura portuária. De modo análogo, pode-se utilizar tais indicadores em canteiros de outros tipos de obras, quer no controle dos processos de gestão, na predição de resíduos, ou em auditorias de sistemas de gestão de resíduos.

\section{Agradecimentos}

Os autores agradecem à Porto Construtora e ao Conselho Nacional de Desenvolvimento Científico e Tecnológico - CNPq o suporte financeiro à pesquisa.

\section{Referências}

ASSOCIAÇÃO BRASILEIRA DE NORMAS TÉCNICAS. NBR 10004: Resíduos Sólidos- 
classificação. Rio de Janeiro, 2004.

BETTS, M. et al. Global construction 2020: a global forecast for the construction industry over the next decade to 2020. London: Global Construction Perspectives and Oxford Economics, 2011.

DE MELO, A. B.; GONÇALVES, A. F.; I. M. MARTINS. Construction and demolition waste generation and management in Lisbon (Portugal). Resources, Conservation and Recycling, v. 55, n. 12, p. 1252-1264, 2011.

LU, W. et al. Estimating and calibrating the amount of building-related construction and demolition waste in urban China. Int. J. Constr. Manag., v.17, n.1, p.13-24, 2017.

NAGALLI, André. Gerenciamento de resíduos na construção civil. São Paulo: Oficina de Textos, 2014.

ONAT, N. C.; KUCUKVAR, M. Carbon footprint of construction industry: A global review and supply chain analysis. Renewable and Sustainable Energy Reviews, v.124, mar.2020. DOI: 10.1016/j.rser.2020.109783.

PAZ, D. H. F. Desenvolvimento de um sistema de apoio à gestão integrada de resíduos sólidos da construção e demolição. 2019. Tese (Doutorado em Engenharia Civil)- Programa de Pós-Graduação em Engenharia Civil, Universidade Federal de Pernambuco, Recife, 2019.

SCHAMNE, A. N.; NAGALLI, A. Evaluation of the potential application of the precepts of solid waste reverse logistics to the civil construction sector in Curitiba, Paraná. International Journal of Environment and Waste Management (Print), v. 22, n. 1-4, p. 24, 2018. DOI: https://doi.org/10.1504/IJEWM.2018.094102.

SILVA M. C.; SANTOS, G. O. Densidade aparente de resíduos sólidos recém coletados. Fortaleza: Instituto Federal de Educação, Ciência e Tecnologia do Ceará, 2011.

VASCONCELOS, K. B.; LEMOS, C. F. Densidade aparente dos resíduos da construção civil em Belo Horizonte - MG. In: CONGRESSO BRASILEIRO DE GESTÃO AMBIENTAL, 6. 2015, Porto Alegre. Anais [...]. Porto Alegre: Centro Universitário Metodista IPA, 2015.

YUAN, H. Key indicators for assessing the effectiveness of waste management in construction projects. Ecolog. Ind., v.24, p. 476-484, 2013. 